\title{
Sozio-technische Existenzbedingungen der Stadt
}

Rezension zu Michael Flinter, Julia Lossau und Anna-Lisa Müller (Hg.) (2017):

Infrastrukturen der Stadt. Wiesbaden: Springer VS.

Abb. 1 Titelseite des Buches (Quelle: Verlag Springer VS)

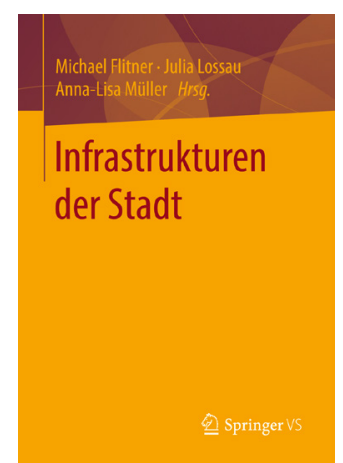

Städte stellen sich heute nicht nur als örtliche Bezugspunkte, kulturelle Formen oder soziale Ökosysteme dar, sondern vornehmend auch als komplexe technische Akteurskonstellationen einer „post-sozialen“ Lebensart (Knorr-Cetina 1997). Infrastrukturen bilden in einer „planetarisch“ verstädterten Lebensform (Brenner/Schmid 2011) einen entscheidenden - vielleicht den entscheidenden - Modus, in dem sich das komplexe Zusammenspiel verschiedener urbaner Akteure zeigt. Dem Sammelband der Bremer Geograph_innen Michael Flitner, Julia Lossau und Anna-Lisa Müller kommt das große Verdienst zu, Stadt und Infrastruktur zusammenzudenken und zu fragen: Welche Heuristiken können uns dabei helfen, die gesellschaftliche Komplexität zu erkunden, die sich aus dem Zusammenspiel des Städtischen mit seinen technisch-infrastrukturellen Bedingungen ergibt?

Die Herausgeber_innen stellen verschiedene methodologische Ansätze und sozialtheoretische Schwerpunkte nebeneinander und versuchen dabei möglichst die Weite des Untersuchungsfeldes zu erfassen, ohne die Kohäsion der Einzelbeiträge des Bandes insgesamt zu gefährden. Das Buch ist in fünf Themenfelder (Leitbilder, Politik, Praktiken, Atmosphären, Regulierungen) mit je zwei Beiträgen unterteilt, die von einer Coda zu „Infrastrukturen im Dorf" ergänzt werden.

In der Einleitung positionieren die Herausgeber_innen ihren Band explizit an der Schnittstelle verschiedener Forschungstraditionen. Insgesamt nimmt der Band aber eine kulturgeographische Perspektive ein, wobei der Einfluss der Science and Technology Studies (STS) mit ihrem Fokus auf die materiellen und technischen Konstitutionsbedingungen gegenwärtiger Gesellschaften besonders auffällig ist. Neben den Beiträgen, die sich mit dem alltagspraktischen Umgang mit Infrastrukturen befassen (Marquardt, Felgenhauer, Müller), sind vor allem Beiträge zu historischen (van Laak, Gandy) und aktuellen Diskursen zu Stadtentwicklung und -planung (Flitner, Beveridge/Naumann, Lossau, Monstadt/Wolff) prominent vertreten. Als gesellschaftstheoretische Rahmenbedingungen heben die Herausgeber_innen zudem den Befund des „splintering urbanism“ (Graham/Marvin 2001) sowie die wachsende Bedeutung sogenannter „kritischer Infrastrukturen“ (Folkers 2012) hervor. Im 
Folgenden werde ich auf die fünf Themenfelder eingehen und jeweils einen Beitrag hervorheben und diskutieren, welche heuristische Impulse dieser der empirischen Forschung zu urbanen Infrastrukturen geben kann.

Im ersten Themenfeld zu „Leitbildern“ städtischer Infrastrukturentwicklung gibt Antje Matern einen Überblick über die historische Koevolution von Städten, technischen Infrastrukturen und ihren kulturellen Leitbildern von Haussmann bis in die Spätmoderne. Michael Flitner fokussiert spezifisch auf das Leitbild „grüner Infrastrukturen“, worunter er nicht Technologien (z. B. Windräder) sondern die belebte Umwelt (z. B. Grünflächen) fasst. Er verweist dabei darauf, dass dieses Konzept sowohl in der Theorie als auch in der politischen Praxis an Aufmerksamkeit gewonnen habe. Diskurskritisch erläutert er, wie Natur hier vor allem nach dem Kriterium ihrer kapitalistischen Verwertbarkeit betrachtet wird. Eine so als allgemeine Produktionsbedingung verstandene Umwelt müsse immer wieder in Bezug auf Maßstäbe (scales) und Quantifizierung formiert und in einen Verwertungszusammenhang eingegliedert werden. Flitner betont, dass städtische Naturen „im Plural“ (Flitner 2017: 60) gedacht und der Kontroverse geöffnet werden müssen, um es Akteur_innen zu erlauben, deren Aneignung lokal zu artikulieren und selbst verschiedene Werte (z. B. Gesundheit, Sicherheit, Freizeitnutzung) zu priorisieren.

Im Feld „Politik“ beschreiben Ross Beveridge und Matthias Naumann den Verlauf der Konflikte zur Energie- und Wasserversorgung in Berlin. Dieser in anderer Fassung schon 2015 erschienene Beitrag scheint allerdings etwas gealtert zu sein. Gerade die der Partei „Die LINKE“ gegenüber sehr kritische Haltung könnte noch erweitert beziehungsweise neu bewertet werden, seitdem diese 2016 erneut Regierungsverantwortung in der Stadt übernommen hat. Nadine Marquards Beitrag zu „Zonen infrastruktureller Entkopplung“ wendet sich von einer Perspektive der STS eher den Mikro-Praktiken zu, die zur permanenten Aushandlung der Vollzugsrealität von Infrastrukturen beitragen. Sie stellt sich explizit gegen die Annahme, Infrastrukturen seien stets unsichtbar und automatisiert. Vielmehr zeigt Marquard beispielhaft anhand von Alltagspraktiken Obdachloser, wie Neu- und Querverbindungen innerhalb infrastruktureller Systeme „bastelnd“ neu geschaffen werden. Zwar bleiben empirisch noch einige Fragen offen: Kann beispielsweise wirklich behauptet werden, dass der illegale Wiederverkauf gebrauchter Tickets für den ÖPNV, die sonst von Nutzer_innen weggeworfen worden wären, in einem relevanten Ausmaß „öffentliche Mobilitätsstrukturen“ schafft, die „,vornehmlich von Armen für Arme organisiert" sind (Marquardt 2017: 96)? Die Anbindung solcher empirisch behandelbarer Fragen an den im Band angeführten Diskussionsstand und die konzeptionelle Einarbeitung der Theorien Manuel Castells' und Michel Serres' eröffnen aber weitgehende forschungspraktische Konsequenzen. So könnten an diesen Beitrag anschließend reflexive - sogar subversive - Bezugnahmen auf Infrastrukturen entwickelt werden oder aber multiperspektivisch die Existenz verschiedener „Sinnschichten“ (Schütz 1971) mit spezifischen Alltagspraktiken als Bedingung städtischer und technischer Umwelten diskutiert werden. Im Vergleich zum Beitrag von Beveridge und Naumann gliedert sich Marquards Verständnis der politischen Aufgeladenheit von Alltagspraktiken auch konzeptionell natürlicher in die Themen des Sammelbandes ein. 
Anna-Lisa Müllers Beitrag zu „Praktiken“ involviert die Leser_innen erneut in eine längere konzeptionelle Diskussion zum Status materieller Agenten in den STS, der womöglich früher im Band besser gepasst hätte. Auch empirische Beispiele wie die Hamburger Müllereimer mit Klappen und die Einführung von Ringbechern können ihre konzeptionellen Punkte zwar gut illustrieren, werden den mit den STS vertrauten Leser_innen jedoch kaum neu erscheinen. Tilo Felgenhauer nähert sich dem Thema digitale Infrastrukturen hingegen mit Blick auf eine systematisch Beforschung. Er lädt dazu ein, der Vorstellung von der Digitalisierung als Enträumlichung zu widerstehen und sie stattdessen als Möglichkeit für kreative und potentiell politische Gestaltung von Sozial- und Raumbeziehungen zu sehen. Felgenhauer beschreibt sich wandelnde Interaktionsformen mit Infrastruktursystemen, die sich auf drei „Dimensionen des Urbanen“ - Rationalisierung, Individualisierung und Vergemeinschaftung - auswirken würden und je spezifische Raumordnungen mit sich bringen: Algorithmen (Rationalisierung) kodieren Räume numerisch, nutzerzentrierte Interface-Gestaltungen (Individualisierung) schaffen ego- und aufenthaltsbezogene Raumbezüge mittels „,indexikalischer“ Sprache (hier, dort, links, rechts) sowie eine von Expert_innenwissen unabhängige Anschlussfähigkeit für Laien (Vergemeinschaftung). Felgenhauer verschiebt so die Perspektive von der machtzentrierten Gegenüberstellung von Produzent und Konsument technischer Infrastrukturen hin zu einer komplexeren Betonung alltäglicher Aneignungsprozesse. Der Fokus liegt auf den Laiennutzer_innen, die über den technischen und alltagssprachlichen Umgang mit digitalen Techniken das Verhältnis von Räumlichkeit und Sozialisierungsformen bestimmen. An Michel Foucault geschulte Leser_innen mögen derartige Schlussfolgerungen wohl wieder machtkritisch einfangen wollen. Felgenhauers sich selbst als „(p)hänomenologisch“ (2017: 121) verstehender Beitrag eröffnet einen auch für diesen Sammelband durchaus eigenständigen Zugang, dem es durch sein konzeptionelles Gerüst der „Dimensionen des Urbanen“ gelingt, der Beforschung von Alltagspraktiken ein heuristisches Raster zu geben. Damit bringt er nicht nur Veränderungen von Interaktionsformen zusammen, sondern bietet auch ein Handwerkszeug für darauf in Zukunft aufbauende Diskussionen, wie es Fallstudien sonst eher selten gelingt.

Im Themenbereich „Atmosphären“ nimmt sich Julia Lossau den Begriff des „Unheimlichen“ vor und versucht dessen heuristischen Nutzen anhand der Analyse eines Entwurfsbeispiels für ein unterirdisches Tunnelnetz in Amsterdam darzustellen. Daran zeige sich eine schon aus Science FictionKlassikern bekannte Dualität der Ermächtigung und Entfremdung durch Technik. Dirk Laak geht weniger vom Konzept einer Atmosphäre aus, sondern vielmehr vom Objekt und zeichnet nach, wie die Stadtstraße kultureller Bezugspunkt in Metropolen und der sich ausbildenden Stadtsoziologie wurde. Der von Georg Simmel, Walter Benjamin und anderen beschriebenen psycho-sozialen Herausforderung der Menschen durch die Stadt wird bereits am Ende des 19. Jahrhunderts mit Verhaltenslehren für die Stadtstraße begegnet. Laak zeigt, wie Infrastrukturen Anlass und Triebfeder reformerischer Erziehung und Disziplinierung „,von oben“ sind, deren spezifische Probleme sowohl technisch (Verkehrsampeln) als auch rechtlich (StVO) gelöst werden. Gleichzeitig werden sie aber auch „von unten“ herausgefordert und 
somit „zum täglichen Test- und Übungsfeld für die zirkulativ strukturierte Gesellschaft der Gegenwart“" (Laak 2017: 157). Diese historische Perspektive ließe sich auf diese Weise auch an andere Formen praxisbasierter Forschung anschließen, zum Beispiel Studien des Typs von Marquardt in diesem Band.

Der fünfte Teil des Bandes, welcher sich dem Thema „Regulierungen“ widmet, ist meines Erachtens der insgesamt gelungenste. Dies liegt zum einen an der Verbindbarkeit beider Beiträge: Sowohl Matthew Gandy als auch Jochen Monstadt und Annika Wolff widmen sich dem Umgang mit Wasser bzw. Energie- und Wasserversorgung in Los Angeles. Zum anderen gelingt es Gandy die Kontingenz und gegenwärtige Unklarheit der Grenze zwischen Naturbedingungen und technischen Infrastrukturen besonders deutlich nachzuzeichnen. Sein historisches Beispiel ist dabei der Los Angeles River, welcher im 19. Jahrhundert noch zur Bewässerung genutzt, nach der Fertigstellung des Los Angeles Aquädukts aber vor allem für den Abwasserabfluss und als Überschwemmungsgebiet eingesetzt wurde. Ab 1935, also während der Zeiten des New Deal, werden großflächig Kanäle errichtet, um den Fluss darin zu unterstützen, wodurch er letztlich als Fluss unkenntlich gemacht wird. Die zunehmende Automobilisierung erhöht den Druck auf das Überschwemmungsgebiet, doch, so zeigt Gandy, wird seit den 1980er Jahren der Fluss Objekt neuer politischer Anrufungen: Ganz verschiedene Organisationen fordern nun eine neue Politik, die sich von der technokratischen Vision der 1930ern unterscheidet. Gandy beschreibt eindrucksvoll die Vielschichtigkeit dieser politischen Projektion und ihrer diversen Leitformeln (technisches Gelingen, soziale Gerechtigkeit, Ökologie).

Resümierend lässt sich feststellen, dass die Stärke des Bandes über die „Infrastrukturen der Stadt“ darin liegt, verschiedene empirisch-qualitative Ansätze aufzuzeigen und Sozialwissenschaftler_innen verschiedener Hintergründe zu ermuntern, Perspektiven auf die Schnittstelle von Urbanität und Infrastruktursystemen zu entwickeln. Angesichts der Vielseitigkeit, der Hybridität und der vermeintlichen Unsichtbarkeit von Infrastrukturen ist ein so facettenreicher Blick fraglos notwendig. Dabei darf auch verziehen werden, dass die Beiträge etwas unvermittelt nebeneinander stehen und eine Beschränkung auf weniger Themenschwerpunkte oder eine stärkere inhaltliche Zentrierung insgesamt gut getan hätte. Beispielsweise wäre auch eine Einteilung in konzeptionelle, diskurs- und planungszentrierte, und auf Alltagspraktiken abzielende Beiträge denkbar gewesen. Als Leser_in ist man im Anschluss an die Lektüre weiterhin mit der Frage konfrontiert, wie viel Orientierungskraft der Zugriff auf „Infrastrukturen der Stadt“" wirklich geben kann. Es lässt sich des Eindrucks nicht ganz verwehren, dass hier zwei Begriffe zusammengebracht werden, die eigenständig bereits kontrovers diskutiert werden. Gemeinsam lassen sie zwar viel Spielraum, aber vielleicht zu viel Spielraum, um daraus einen letztlich kohärenten Sammelband zu gestalten. Hervorzuheben sind jene Beiträge, für die es nicht um Infrastrukturen in der Stadt geht, bei denen also das Verhältnis von Stadt und Infrastuktur nicht relativ zufällig wirkt, sondern denen es gelingt, die hybride und sich wandelnde Gestalt des Städtischen durch seine Infrastrukturen zu erzählen (ich denke da vor allem an die Beiträge von Marquardt, Felgenhauer, van Laak, Lossau und Gandy).

Zudem lässt sich sagen, dass der Sammelband trotz seiner Breite einen Bezug zum globalen Süden vermissen lässt, wie die Herausgeber_innen selbst 
eingestehen (Flittner/Lossa/Müller 217: 15). Im Lichte der Herausforderung nicht-okzidentaler Megacities erscheint dies jedoch durchaus als verpasste Chance. Auch stärker feministische Perspektiven fehlen, was angesichts der vielen Bezüge auf die STS etwas verwundert. Es ließe sich im Anschluss an die feministische Technikforschung vermutlich doch einiges über die (Un-)Sichtbarkeiten von Infrastrukturen und infrastruktureller Arbeit sagen. Zugegebenermaßen würde dies das unterliegende Problem, die vielen Beiträge in eine kohärentere Form zu bringen, jedoch nur noch vergrößern.

Von besonderem Interesse wird dieser Band für handlungstheoretisch, mikrosoziologisch und an den STS interessierte Leser_innen sein. Für künftige Projekte wäre ein stärker thematisch fokussierter Zuschnitt zu wünschen, den dieser Sammelband wohl noch nicht leisten kann. Doch ist es mit dessen Hilfe vielleicht möglich, sich an einzelnen Beiträgen zu orientieren und daraus eine eigenständige und stärker geschlossene Perspektive zu entwickeln. Für Geograph_innen, Soziolog_innen und gerade auch Studierende in der Explorationsphase bietet dieser Sammelband dafür reichliche Forschungsbeispiele und Inspiration in einem Themenfeld, dessen Bedeutung zweifellos weiterhin wachsen wird.

\section{Autor_innen}

Johannes Coughlan ist Soziologe und forscht aktuell praxeographisch zu Bewertungskriterien in Entwurfspraktiken der Architektur.

johannescoughlan@gmail.com

\section{Literatur}

Brenner, Neil / Schmid, Christian (2011): Planetary urbanisation. In: Matthew Gandy (Hg.), Urban Constellations. Berlin: Jovis, 10-13.

Knorr-Cetina, Karin (1997): Sociality with objects. Social relations in postsocial knowledge societies. In: Theory, Culture \& Society 14/4, 1-30.

Felgenhauer, Tilo (2017): Die Räume des Codes und die Räume des Alltags - Zur Aneignung urbaner digitaler Infrastrukturen. In: Michael Flitner / Julia Lossau / Anna-Lisa Müller (Hg.), Infrastrukturen der Stadt. Wiesbaden: Springer VS, 107-124.

Flitner, Michael (2017): Grüne Infrastruktur und die Erneuerung städtischer Naturen. In: Michael Flitner / Julia Lossau / Anna-Lisa Müller (Hg.), Infrastrukturen der Stadt. Wiesbaden: Springer VS, 45-64.

Flitner, Michael / Lossa, Julia / Müller, Anna-Lisa (2017): Infrastruktur, Stadt und Gesellschaft. Eine Einleitung. In: Michael Flitner / Julia Lossau / Anna-Lisa Müller (Hg.), Infrastrukturen der Stadt. Wiesbaden: Springer VS, 1-19.

Folkers, Andreas (2012): Kritische Infrastruktur. In: Nadine Marquardt / Verena Schreiber (Hg.), Ortsregister. Ein Glossar zu Räumen der Gegenwart. Bielefeld: transcript, 154-159.

Graham, Steve / Marvin, Simon (2001): Splintering Urbanism. Networked Infrastructures, Technological Mobilities and the Urban Condition. London/New York: Routledge.

Laak, Dirk (2017): Vom Lebensraum zum Leitungsweg. Die Stadtstraße als soziale Arena. In: Michael Flitner / Julia Lossau / Anna-Lisa Müller (Hg.) (2017), Infrastrukturen der Stadt. Wiesbaden: VS, 145-162.

Marquardt, Nadine (2017): Zonen infrastruktureller Entkopplung. Urbane Prekarität und soziotechnische Verknüpfungen im öffentlichen Raum. In: Michael Flitner / Julia Lossau / Anna-Lisa Müller (Hg.), Infrastrukturen der Stadt. Wiesbaden: Springer VS, 89-104.

Schütz, Alfred (1971): Über die Mannigfaltigen Wirklichkeiten. In: Gesammelte Aufsätze. Dordrecht: Springer, 237-298. 
